\title{
Discrete approximation of quantum stochastic models
}

\author{
Luc Bouten ${ }^{1, a)}$ and Ramon Van Handel ${ }^{2, b)}$ \\ ${ }^{1}$ Physical Measurement and Control 266-33, California Institute of Technology, Pasadena, \\ California 91125, USA \\ ${ }^{2}$ Department of Operations Research and Financial Engineering, Princeton University, \\ Princeton, New Jersey 08544, USA
}

(Received 16 July 2008; accepted 26 September 2008; published online 20 October 2008)

\begin{abstract}
We develop a general technique for proving convergence of repeated quantum interactions to the solution of a quantum stochastic differential equation. The wide applicability of the method is illustrated in a variety of examples. Our main theorem, which is based on the Trotter-Kato theorem, is not restricted to a specific noise model and does not require boundedness of the limit coefficients. (C) 2008 American Institute of Physics. [DOI: 10.1063/1.3001109]
\end{abstract}

\section{INTRODUCTION}

It has been well established that the quantum stochastic equations introduced by Hudson and Parthasarathy ${ }^{18}$ provide an essential tool in the theoretical description of physical systems, especially those arising in quantum optics. The time evolution in these models is given by a unitary cocycle that solves a Hudson-Parthasarathy quantum stochastic differential equation (QSDE). These unitaries define a flow, which is a quantum Markov process in the sense of Ref. 1, that represents the Heisenberg time evolution of the observables of the physical system. Several authors have studied how quantum stochastic models can be obtained as a limit of fundamental models in quantum field theory. ${ }^{2,15,9}$ This provides a sound justification for using quantum stochastic models to describe physical systems arising, e.g., in quantum optics.

In contrast to the limit theorems for field theoretic models, which are non-Markovian and have continuous time parameter, it is natural to ask whether QSDEs can be obtained as a limit of discrete time quantum Markov chains. Classical counterparts of such results are ubiquitous in probability theory and there is a variety of motivations (to be discussed further below) to study such limits. The first results on this topic date back to the work of Lindsay and Parthasarathy. ${ }^{26,21}$ In Ref. 26 it is shown that a particular class of repeated interaction models, where a physical system is coupled to a spin chain, converge in a very weak sense (in matrix elements) to the solution of a QSDE. A significant step forward was taken in Ref. 21 where Lindsay and Parthasarathy embedded a chain of finite-dimensional noise systems in the algebra of bounded operators on the Fock space and showed strong convergence of the discrete flow to the flow obtained from a QSDE. Much later, Attal and Pautrat ${ }^{3}$ obtained similar results (in the special case of a spin chain) by showing that the discrete unitaries (rather than the flows) converge strongly to the solution of a QSDE.

Independent of the previous work on the convergence of discrete chains, Holevo ${ }^{16,17}$ studied a very similar problem in his work on time-ordered exponentials in quantum stochastic calculus. The essence of Holevo's approach is to define time-ordered stochastic exponentials as the limit of discrete interaction models, where the role of the discrete noise is played by the increments in the field operators (the discrete noise is thus infinite dimensional in this setting, in contrast to the

\footnotetext{
${ }^{a)}$ Electronic mail: bouten@its.caltech.edu.

${ }^{b)}$ Electronic mail: rvan@princeton.edu.
} 
finite-dimensional models considered by Lindsay and Parthasarathy ${ }^{21}$ ). Despite the rather different motivation, the results of Holevo ${ }^{16,17}$ are strikingly similar to those obtained in the study of limits of discrete interaction models, as has been pointed out by Gough. ${ }^{14}$

None of these results, however, are capable of dealing with the physically important case of limit QSDEs with unbounded initial coefficients (a typical setting in, e.g., quantum optics). This restriction is inherent to the techniques used to prove these results, which rely on Dyson series expansions and require boundedness of the coefficients. Moreover, each of the above results has been proved separately in its own setting, while the similarity between these results strongly suggests that they should be unified within a common framework.

The purpose of this paper is to introduce a general technique for proving convergence of a sequence of discrete quantum Markov chains to the solution of a QSDE. Our approach does not rely on a Dyson series expansion but instead employs a form of the Trotter-Kato theorem. This allows us to deal with unbounded coefficients in a natural and transparent manner. In the simpler case where the limit coefficients and/or discrete noises are bounded, we obtain many of the previous results as special cases of our general theorems. Moreover, the specific functional form of the limiting coefficients, obtained in Refs. 3, 16, and 14 by identifying a power series obtained from the Dyson expansion, is effectively demystified: we will see that it is an immediate consequence of the scaling of the noise operators.

Our motivation for this work is twofold. First, the convergence of discrete quantum Markov chains to continuous ones is a fundamental problem in quantum probability theory. In classical probability theory such problems have been investigated for many decades, and the theory has culminated in the well known work of Stroock and Varadhan (Ref. 30, Sec. 11.2), Ethier and Kurtz, ${ }^{10}$ and Kushner, ${ }^{20}$ among others. A similar systematic investigation was hitherto lacking in the quantum probability literature. This paper presents one attempt to unify and extend the existing results in this direction.

Second, we are motivated by practical problems in which one is specifically interested in the convergence of discrete to continuous models. For example, certain laboratory experiments, e.g., atomic beam experiments with a large flux of atoms, can be approximately modeled by quantum stochastic equations in the limit of large flux. Another application of independent interest is the development of numerical methods for quantum stochastic models. To perform tractable numerical simulations, one is often forced to discretize, particularly in dynamical optimization problems which appear in the emerging field of quantum engineering, ${ }^{6}$ and convergence of the discretized approximations is a challenging topic. We were motivated in particular by the problem of discretizing linear quantum systems, which play a special role in linear systems theory, ${ }^{19,25,24}$ but are not covered by previous results as both the noise and the initial system are necessarily unbounded.

As compared to previous results, our approach is closest to the original method of Lindsay and Parathasarathy. ${ }^{21}$ The simple uniform convergence result (Ref. 21, Proposition 3.3) is replaced in our setting by a variant of the Trotter-Kato theorem, ${ }^{10}$ which allows us to deal with the analytic complications inherent to the case of unbounded coefficients. We also work directly with the unitary evolution rather than with the flow. The Trotter-Kato theorem allows us to obtain convergence by studying generators and exploits in a fundamental way the Markov property of both the approximate and the limit evolutions.

Techniques to obtain convergence for QSDEs by studying generators were introduced by Fagnola $^{12}$ and Chebotarev $^{8}$ (using resolvents) and by Lindsay and Wills ${ }^{22,23}$ (using the TrotterKato theorem). We have previously applied related techniques to obtain general results on singular perturbation problems for quantum stochastic models. ${ }^{5,7}$ The application of this type of technique to obtain the convergence of discrete quantum models is new.

The remainder of this paper is organized as follows. In Sec. II we introduce the class of discrete interaction models and limit models which will be of interest throughout the paper, and we state our main results. The main theorem is a generalization of the Trotter-Kato theorem to quantum stochastic models and is generally applicable. We also introduce a more restricted family 
of discrete models for which the conditions of this result can be verified explicitly. Section III develops a variety of known and new examples using our results. Finally, Sec. IV is devoted to the proofs of our results.

\section{MAIN RESULTS}

In Secs. II A-II D, we first define the class of models that we will consider and introduce the necessary assumptions. This is followed by the statement of our main results. The proofs of our main results are contained in Sec. IV below.

\section{A. The limit model}

Throughout this paper we let $\mathcal{H}$, the initial space, be a separable (complex) Hilbert space. We denote by $\mathcal{F}=\Gamma_{s}\left(L^{2}\left(\mathbb{R}_{+} ; C^{n}\right)\right)$ the symmetric Fock space with multiplicity $n \in \mathbb{N}$ [i.e., the oneparticle space is $\left.\mathrm{C}^{n} \otimes L^{2}\left(\mathrm{R}_{+}\right) \cong L^{2}\left(\mathrm{R}_{+} ; \mathrm{C}^{n}\right)\right]$ and by $e(f), f \in L^{2}\left(\mathbb{R}_{+} ; \mathbb{C}^{n}\right)$ the exponential vectors in $\mathcal{F}$. The annihilation, creation, and gauge processes on $\mathcal{F}$, as well as their ampliations to $\mathcal{H} \otimes \mathcal{F}$, will be denoted as $A_{t}^{i}, A_{t}^{i \dagger}$, and $\Lambda_{t}^{i j}$, respectively (the channel indices are relative to the canonical basis of $\mathbb{C}_{1}^{n}$ ). Moreover, we will fix once and for all a dense domain $\mathcal{D} \subset \mathcal{H}$ and a dense domain of exponential vectors $\mathcal{E}=\operatorname{span}\{e(f): f \in \mathfrak{S}\} \subset \mathcal{F}$, where $\mathfrak{S} \subset L^{2}\left(\mathbb{R}_{+} ; \mathbb{C}^{n}\right) \cap L_{\mathrm{loc}}^{\infty}\left(\mathbb{R}_{+} ; \mathbb{C}^{n}\right)$ is an admissible subspace in the sense of Hudson-Parthasarathy ${ }^{18}$ which is presumed to contain at least all simple functions. An introduction to these concepts (using a similar notation to the one used here) can be found in Ref. 4 and we refer to Refs. 18 and 27 for a detailed description of quantum stochastic calculus.

Consider a QSDE of the form

$$
d U_{t}=U_{t}\left\{\sum_{i, j=1}^{n}\left(N_{i j}-\delta_{i j}\right) d \Lambda_{t}^{i j}+\sum_{i=1}^{n} M_{i} d A_{t}^{i \dagger}+\sum_{i=1}^{n} L_{i} d A_{t}^{i}+K d t\right\},
$$

where $U_{0}=I$ and the quantum stochastic integrals are defined relative to the domain $\mathcal{D} \otimes \mathcal{E}$ (for simplicity, we will use the same notation for operators on $\mathcal{H}$ or on $\mathcal{F}$ and for their ampliations to $\mathcal{H} \otimes \mathcal{F}$ ). Under certain conditions to be introduced below, the solution of this equation describes the time evolution of quantum stochastic models such as those used in quantum optics. The purpose of this paper is to prove that the solution of this equation may be approximated by appropriately chosen discrete interaction models. The approximating models will be defined in Sec. II B.

Denote by $\theta_{t}: L^{2}\left(\left[t, \infty\left[; \mathbb{C}^{n}\right) \rightarrow L^{2}\left(\mathbb{R}_{+} ; \mathbb{C}^{n}\right)\right.\right.$ the canonical shift $\theta_{t} f(s)=f(t+s)$ and by $\Theta_{t}: \mathcal{F}_{[t}$ $\rightarrow \mathcal{F}$ its second quantization (here $\mathcal{F} \cong \mathcal{F}_{t]} \otimes \mathcal{F}_{[t}$ denotes the usual continuous tensor product decomposition). Recall that an adapted process $\left\{U_{t}: t \geq 0\right\}$ on $\mathcal{H} \otimes \mathcal{F}$ is called a unitary cocycle if $U_{t}$ is unitary for all $t \geq 0, t \mapsto U_{t}$ is strongly continuous, and $U_{s+t}=U_{s}\left(I \otimes \Theta_{s}^{*} U_{t} \Theta_{s}\right)$, where $I$ $\otimes \Theta_{s}^{*} U_{t} \Theta_{s}$ is viewed as an operator on $\mathcal{F}_{s]} \otimes\left(\mathcal{H} \otimes \mathcal{F}_{[s}\right) \cong \mathcal{H} \otimes \mathcal{F}$. The following condition will always be presumed to be in force.

Condition 1: The operators $K, L_{i}, M_{i}$, and $N_{i j}$, defined on the domain $\mathcal{D}$, are such that the Hudson-Parthasarathy equation (1) possesses a unique solution $\left\{U_{t}: t \geq 0\right\}$ which extends to a unitary cocycle on $\mathcal{H} \otimes \mathcal{F}$.

Remark 1: When the coefficients $K, L_{i}, M_{i}$, and $N_{i j}$ are bounded, it is well known ${ }^{18}$ that Condition 1 holds true if and only if the following algebraic relations are satisfied:

$$
K+K^{*}=-\sum_{i=1}^{n} L_{i} L_{i}^{*}, \quad M_{i}=-\sum_{j=1}^{n} N_{i j} L_{j}^{*}
$$




$$
\sum_{j=1}^{n} N_{m j} N_{\ell j}^{*}=\sum_{j=1}^{n} N_{j m}^{*} N_{j \ell}=\delta_{m \ell} .
$$

Remarks on the verification of Condition 1 in the unbounded case can be found in Ref. 7.

Remark 2: We have chosen the left Hudson-Parthasarathy equation (1) rather than the more familiar right equation where the solution is placed to the right of the coefficients. This means that the Schrödinger evolution of a state vector $\psi \in \mathcal{H} \otimes \mathcal{F}$ is given by $U_{t}^{*} \psi$, etc. The reason for this choice is that for equations with unbounded coefficients, it is generally much easier to prove the existence of a unique cocycle solution for the left equation than for the right equation (see, e.g., Refs. 11 and 22). As we will ultimately prove convergence of discrete evolutions to $U_{t}^{*}$, there is no loss of generality in working with the more tractable left equations. If we wish to begin with a well defined right equation (as is more natural when the coefficients are bounded), our results can be immediately applied to the Hudson-Parthasarathy equation for its adjoint.

\section{B. The discrete approximations}

A discrete interaction model describes the repeated interaction of an initial system with independent copies of an external noise source. Given an initial Hilbert space $\mathcal{H}^{\prime}$ and a noise Hilbert space $\mathcal{K}^{\prime}$, a single interaction is described by a unitary operator $R^{\prime}$ on $\mathcal{H}^{\prime} \otimes \mathcal{K}^{\prime}$. To describe repeated interactions, we work on the Hilbert space $\mathcal{H}^{\prime} \otimes \otimes_{\mathrm{N}} \mathcal{K}^{\prime}$ on which we define the natural isomorphism,

$$
\Xi_{k}:\left(\mathcal{K}^{\prime}\right)^{\otimes(k-1)} \otimes \mathcal{H}^{\prime} \otimes \otimes \mathcal{K}^{\prime} \rightarrow \mathcal{H}^{\prime} \otimes \otimes \mathcal{K}^{\prime},
$$

as $\Xi_{k}\left(\psi_{k-1]} \otimes \xi \otimes \psi_{[k}\right)=\xi \otimes\left(\psi_{k-1]} \otimes \psi_{[k}\right)$. We now define recursively $R_{0}^{\prime}=I$ and

$$
R_{k}^{\prime}\left(\xi \otimes \psi_{k-1]} \otimes \psi_{k} \otimes \psi_{[k+1}\right)=R_{k-1}^{\prime} \Xi_{k}\left(\psi_{k-1]} \otimes R^{\prime}\left(\xi \otimes \psi_{k}\right) \otimes \psi_{[k+1}\right)
$$

for $k \in \mathbb{N}$, where $\psi_{k-1]} \otimes \psi_{k} \otimes \psi_{[k+1} \in\left(\mathcal{K}^{\prime}\right)^{\otimes(k-1)} \otimes \mathcal{K}^{\prime} \otimes \otimes_{\mathrm{N}} \mathcal{K}^{\prime} \cong \otimes_{\mathrm{N}} \mathcal{K}^{\prime}$ and $\xi \in \mathcal{H}^{\prime}$. This is precisely the discrete counterpart of a unitary cocycle. Note that the order of multiplication in the recursion for $R_{k}^{\prime}$ matches the choice of the left Hudson-Parthasarathy equation above (i.e., $R_{k}^{\prime *} \psi$ is the Schrödinger evolution, see Remark 2).

The purpose of this paper is to prove that the solution of the Hudson-Parthasarathy equation (1) can be approximated by a sequence of discrete interaction models with decreasing time step. In order to study this problem, we will embed our discrete interaction models in the limit Hilbert space $\mathcal{H} \otimes \mathcal{F}$. This allows us to prove strong convergence of the embedded discrete cocycles to the solution of Eq. (1). The precise way in which the embedding is done does not affect the proof of our main result; we therefore proceed in a general fashion by defining a fixed but otherwise arbitrary sequence of discrete interaction models which are already embedded into the limit Hilbert space (this avoids, without any loss of generality, the notational burden of introducing separate Hilbert spaces and embedding maps for every discrete approximation). As a special case of this general construction, we will introduce in Sec. II D below an interesting class of discrete interaction models for which the embedding is made explicit.

We proceed to introduce the embedded discrete interaction models. For every $k \in \mathbb{N}$, we will define a discrete interaction model with time $\operatorname{step}^{1} 2^{-k}$ (the ultimate goal being to take the limit as $k \rightarrow \infty)$. By its continuous tensor product property, the Fock space is isomorphic to $\mathcal{F} \cong \bigotimes_{\mathrm{N}} \mathcal{F}_{\left.2^{-k}\right]}$, where each component $\mathcal{F}_{\left.2^{-k}\right]}$ represents a consecutive time slice of length $2^{-k}$. Our discrete interaction models will be embedded as repeated interactions with consecutive time slices

${ }^{1}$ This choice is only made to keep our notation manageable and is by no means a restriction of the method. 
of the field. Let us make this precise: the following notations will be used throughout the paper. Let $\mathcal{H}^{k} \subset \mathcal{H}$ be the initial Hilbert space and let $\mathcal{K}^{k} \subset \mathcal{F}_{\left.2^{-k}\right]}$ be the noise Hilbert space for the interaction model with time step $2^{-k}$. We will write

$$
\mathcal{F}^{k}=\bigotimes_{\mathrm{N}}^{\otimes} \mathcal{K}^{k} \subset \underset{\mathrm{N}}{\otimes} \mathcal{F}_{\left.2^{-k}\right]} \cong \mathcal{F} .
$$

We now introduce a unitary operator $R^{k}$ on $\mathcal{H}^{k} \otimes \mathcal{K}^{k}$ which describes the interaction in a single time step, and we extend $R^{k}$ to $\mathcal{H} \otimes \mathcal{F}$ by setting $R^{k} \psi=\psi$ for $\psi \in\left(\mathcal{H}^{k} \otimes \mathcal{K}^{k} \otimes \mathcal{F}_{\left[2^{-k}\right.}\right)^{\perp}$. We now define recursively $R_{t}^{k}=I$ for $t \in\left[0,2^{-k}[\right.$ and

$$
R_{t}^{k}=R_{(\ell-1) 2^{-k}}^{k}\left(I \otimes \Theta_{(\ell-1) 2^{-k}}^{*} R^{k} \Theta_{(\ell-1) 2^{-k}}\right) \quad \text { for } t \in\left[\ell 2^{-k},(\ell+1) 2^{-k}[, \quad \ell \in \mathbb{N},\right.
$$

where $I \otimes \Theta_{\ell 2^{-k}}^{*} R^{k} \Theta_{\ell 2^{-k}}$ is viewed as an operator on $\mathcal{F}_{\left.\ell 2^{-k}\right]} \otimes\left(\mathcal{H} \otimes \mathcal{F}_{\left[\ell 2^{-k}\right.}\right) \cong \mathcal{H} \otimes \mathcal{F}$. In other words, the discrete evolution $R_{t}^{k}$ is an adapted unitary process which is piecewise constant on consecutive intervals of length $2^{-k}$, and an interaction between the initial system and the next field slice occurs at the beginning of every interval. Our goal is to prove that $R_{t}^{k}$ converges as $k \rightarrow \infty$ to the solution $U_{t}$ of Eq. (1).

Remark 3: This model coincides precisely with the discrete interaction model defined above if we choose $\mathcal{H}^{\prime}=\mathcal{H}^{k}, \mathcal{K}^{\prime}=\mathcal{K}^{k}, R^{\prime}=R^{k}$, and $R_{\ell}^{\prime}=R_{\ell 2-n}^{k}$.

\section{A general limit theorem}

Before we proceed to the statement of our main result, we introduce certain families of semigroups which will play a central role in our approach.

Lemma 1: For $k \in \mathbb{N}$ and $\psi, \varphi \in \mathcal{F}_{\left.2^{-k}\right]}$, define $R^{k ; \psi \varphi}: \mathcal{H} \rightarrow \mathcal{H}$ such that

$$
\left\langle u, R^{k ; \psi \varphi} v\right\rangle=\|\psi\|^{-1}\|\varphi\|^{-1}\left\langle u \otimes \psi, R^{k} v \otimes \varphi\right\rangle \quad \forall u, v \in \mathcal{H} .
$$

Then $R^{k ; \psi \varphi}$ is a contraction on $\mathcal{H}$ and

$$
\left\langle u,\left(R^{k ; \psi \varphi}\right)^{\left[t 2^{k}\right]} v\right\rangle=\|\psi\|^{-N 2^{k}}\|\varphi\|^{-N 2^{k}}\left\langle u \otimes \psi^{\otimes N 2^{k}}, R_{t}^{k} v \otimes \varphi^{\otimes N 2^{k}}\right\rangle
$$

for all $u, v \in \mathcal{H}$ and $t \in[0, N], N \in \mathbb{N}$.

Proof. This follows directly from the unitarity of $R^{k}$ and the definition of $R_{t}^{k}$.

The following counterpart for the limit equation (1) is proved in Ref. 7, Lemma 1.

Lemma 2: For $\alpha, \beta \in \mathbb{C}^{n}$, define $T_{t}^{\alpha \beta}: \mathcal{H} \rightarrow \mathcal{H}$ such that

$$
\left\langle u, T_{t}^{\alpha \beta} v\right\rangle=e^{-\left(|\alpha|^{2}+|\beta|^{2}\right) t / 2}\left\langle u \otimes e\left(\alpha I_{[0, t]}\right), U_{t} v \otimes e\left(\beta I_{[0, t]}\right)\right\rangle \quad \forall u, v \in \mathcal{H}, \quad t \geq 0 .
$$

Then $T_{t}^{\alpha \beta}$ is a strongly continuous contraction semigroup on $\mathcal{H}$, and the generator $\mathcal{L}^{\alpha \beta}$ of this semigroup satisfies $\operatorname{Dom}\left(\mathcal{L}^{\alpha \beta}\right) \supset \mathcal{D}$ such that for $u \in \mathcal{D}$

$$
\mathcal{L}^{\alpha \beta} u=\left(\sum_{i, j=1}^{n} \alpha_{i}^{*} N_{i j} \beta_{j}+\sum_{i=1}^{n} \alpha_{i}^{*} M_{i}+\sum_{i=1}^{n} L_{i} \beta_{i}+K-\frac{|\alpha|^{2}+|\beta|^{2}}{2}\right) u .
$$

The reason to focus on the semigroups associated to our models is that we will seek conditions for convergence of the discrete approximations in terms of the generators $\mathcal{L}^{\alpha \beta}$. As the latter is expressed directly in terms of the coefficients of the limit equation (1), such conditions can typically be verified in a straightforward manner and do not require us to work directly with the solution $U_{t}$ of that equation.

The following is the main result of this paper. The theorem bears a strong resemblance to the Trotter-Kato theorem for contraction semigroups, and the latter does indeed form the foundation of the proof. The proof of the theorem is given in Sec. IV.

Theorem 1: Assume that Condition 1 holds, and let $\mathcal{D}^{\alpha \beta} \subset \operatorname{Dom}\left(\mathcal{L}^{\alpha \beta}\right)$ be a core for $\mathcal{L}^{\alpha \beta}$, $\alpha, \beta \in \mathrm{C}^{n}$. Then the following conditions are equivalent. 
(1) For all $\alpha, \beta \in \mathbb{C}^{n}, u \in \mathcal{D}^{\alpha \beta}$ there exist $u^{k} \in \mathcal{H}$ and $\psi^{k}, \varphi^{k} \in \mathcal{F}_{\left.2^{-k}\right]}$ so that

$$
u^{k} \stackrel{k \rightarrow \infty}{\longrightarrow} u, \quad\left(\psi^{k}\right)^{\otimes 2^{k}} \stackrel{k \rightarrow \infty}{\longrightarrow} e\left(\alpha I_{[0,1]}\right), \quad\left(\varphi^{k}\right)^{\otimes 2^{k}} \stackrel{k \rightarrow \infty}{\longrightarrow} e\left(\beta I_{[0,1]}\right),
$$

and

$$
2^{k}\left(R^{k ; \psi^{k} \varphi^{k}}-I\right) u^{k} \stackrel{k \rightarrow \infty}{\longrightarrow} \mathcal{L}^{\alpha \beta} u
$$

(2) For every $T<\infty$ and $\psi \in \mathcal{H} \otimes \mathcal{F}$

$$
\lim _{k \rightarrow \infty} \sup _{0 \leq t \leq T}\left\|R_{t}^{k *} \psi-U_{t}^{*} \psi\right\|=0
$$

Remark 4: As was pointed out to us by a referee, this theorem could also be stated outside the framework of QSDEs. Indeed, a careful reading of the proof reveals that one may dispose of Condition 1 entirely and assume only that $U_{t}$ is any unitary cocycle on $\mathcal{H} \otimes \mathcal{F}$. The theorem is the most powerful, however, when the generators $\mathcal{L}^{\alpha \beta}$ admit an explicit expression in terms of the parameters of the limit model. This is the case, in particular, when Condition 1 is satisfied and $\mathcal{D}$ is a core for all $\mathcal{L}^{\alpha \beta}, \alpha, \beta \in \mathbb{C}$. We can then choose $\mathcal{D}^{\alpha \beta} \subset \mathcal{D}$, with the important consequence that this puts the explicit expression for $\mathcal{L}^{\alpha \beta}$ in Lemma 2 at our disposal. This will be the case in all our examples. Typically existence and uniqueness proofs for the solution of (1) already imply that $\mathcal{D}$ is a core for $\mathcal{L}^{\alpha \beta}$, see, e.g., Refs. 11, 22, and 7 (Remark 4) for further comments.

Remark 5: The assumption in Condition 1 that $U_{t}$ is a unitary cocycle can be weakened somewhat. In the absence of this assumption, one may still prove the implication $1 \Rightarrow 2$ provided that strong convergence uniformly on compact intervals is replaced by weak convergence of $R_{t}^{k}$ to $U_{t}$ for every time $t$. We have chosen to concentrate on the unitary case, as it is the relevant one for physical applications and admits a much stronger result.

\section{A class of discrete interactions}

Theorem 1 is a very general result which allows us to infer the convergence of a sequence of discrete interaction models to the solution of the Hudson-Parthasarathy equation (1). The verification of the conditions of the theorem requires additional work, however, and the form of the limit coefficients depends on the choice of the discrete interactions $R^{k}$. In this section we introduce a special class of discrete interaction models (with $\mathcal{H}^{k}=\mathcal{H}$ ) for which the conditions of Theorem 1 can be verified explicitly. In particular, we obtain explicit expressions for the limit coefficients. It should be noted that this class of discrete interaction models is physically natural, and we will encounter several concrete examples in Sec. III.

Let $\mathcal{K}$ be a fixed Hilbert space and suppose that we are given a sequence of bounded operators $\pi^{k}: \mathcal{K} \rightarrow \mathcal{F}_{\left.2^{-k}\right]}$ which are partially isometric in the following sense:

$$
\pi^{k *} \pi^{k}=I_{\mathcal{K}}, \quad \pi^{k} \pi^{k *}=P_{\mathcal{K}^{k}} \text { for all } k, \text { where } \mathcal{K}^{k}:=\operatorname{ran} \pi^{k}
$$

Here $P_{\mathcal{K}^{k}}$ is the orthogonal projection onto $\mathcal{K}^{k}$ and $I_{\mathcal{K}}$ is the identity on $\mathcal{K}$. The same space $\mathcal{K}$ will play the role of the noise Hilbert space for every discrete interaction model $R^{k}(k \in \mathbb{N})$ after being isometrically embedded into the limit Hilbert space by the embedding maps $\pi^{k}$. As we will see, the choice to work with a fixed noise space prior to embedding is convenient due to the fact that the limit coefficients can be expressed in terms of the matrix elements of certain operators on $\mathcal{H} \otimes \mathcal{K}$.

We now define the discrete models. Let $F_{1}, \ldots, F_{\ell}, G_{1}, \ldots, G_{m}$, and $H_{1}, \ldots, H_{r}(\ell, m, r \in \mathbb{N})$ be bounded self-adjoint operators on $\mathcal{H}$, and let $\lambda_{1}, \ldots, \lambda_{\ell}, \mu_{1}, \ldots, \mu_{m}$, and $\nu_{1}, \ldots, \nu_{r}$ be (not necessarily bounded) self-adjoint operators on $\mathcal{K}$. Define 


$$
H^{k}=2^{k} \sum_{j=1}^{\ell} F_{j} \otimes \lambda_{j}+2^{k / 2} \sum_{j=1}^{m} G_{j} \otimes \mu_{j}+\sum_{j=1}^{r} H_{j} \otimes \nu_{j}
$$

on $\mathcal{H} \bar{\otimes}\left(\bigcap_{j} \operatorname{Dom}\left(\lambda_{j}\right) \cap \bigcap_{j} \operatorname{Dom}\left(\mu_{j}\right) \cap \bigcap_{j} \operatorname{Dom}\left(\nu_{j}\right)\right):=D_{0}$.

Condition 2: The following are presumed to hold:

(1) $H^{k}$ is essentially self-adjoint for every $k$.

(2) $\quad \check{F}:=F_{1} \otimes \lambda_{1}+\cdots+F_{\ell} \otimes \lambda_{\ell}$ is essentially self-adjoint on the domain $\mathcal{D}_{0}$.

(3) There is a family of orthonormal vectors $\chi_{0}, \ldots, \chi_{n} \in \mathcal{K}$ such that for all $\alpha \in \mathbb{C}^{n}$, the vector $\chi^{k}(\alpha):=\chi_{0}+2^{-k / 2} \sum_{j=1}^{n} \alpha_{j} \chi_{j}$ satisfies $\left(\pi^{k} \chi^{k}(\alpha)\right)^{\otimes 2^{k}} \rightarrow e\left(\alpha I_{[0,1]}\right)$.

(4) $\chi_{0} \in \operatorname{Dom}\left(\nu_{j}\right)$ for all $j$.

(5) $\chi_{0} \in \operatorname{Dom}\left(\mu_{j}\right)$ and $\left\langle\chi_{0}, \mu_{j} \chi_{0}\right\rangle=0$ for all $j$.

(6) $\chi_{0} \in \operatorname{Dom}\left(\lambda_{j}\right)$ and $\lambda_{j} \chi_{0}=0$ for all $j$.

We subsequently identify $H^{k}$ and $\check{F}$ with their closures. We now define the discrete interaction unitary $R^{k}:=\pi^{k} e^{i H^{k} 2^{-k}} \pi^{k *}$ on $\mathcal{H} \otimes \mathcal{K}^{k}$ and extend to $\mathcal{H} \otimes \mathcal{F}$ as usual.

To state the convergence result for this class of discrete interaction models we must introduce the corresponding limit coefficients $N_{i j}, M_{i}, L_{i}$, and $K$ in Eq. (1). This is what we turn to presently. Define the bounded continuous functions $f, g: \mathbb{R} \rightarrow \mathrm{C}$ as

$$
f(x)=\frac{e^{i x}-1}{x}, \quad g(x)=\frac{e^{i x}-i x-1}{x^{2}} .
$$

We must also define the bounded operators $W_{i j}, X_{i}^{p}, Y_{i}^{p}, Z^{p q}(i, j=1, \ldots, m, p, q=1, \ldots, n)$ on $\mathcal{H}$ as follows: for every $u, v \in \mathcal{H}$,

$$
\begin{gathered}
\left\langle u, W_{i j} v\right\rangle=\left\langle u \otimes \mu_{i} \chi_{0}, g(\check{F}) v \otimes \mu_{j} \chi_{0}\right\rangle, \\
\left\langle u, X_{i}^{p} v\right\rangle=\left\langle u \otimes \chi_{p}, f(\check{F}) v \otimes \mu_{i} \chi_{0}\right\rangle, \\
\left\langle u, Y_{i}^{p} v\right\rangle=\left\langle u \otimes \mu_{i} \chi_{0}, f(\check{F}) v \otimes \chi_{p}\right\rangle, \\
\left\langle u, Z^{p q} v\right\rangle=\left\langle u \otimes \chi_{p}, e^{i \check{F}} v \otimes \chi_{q}\right\rangle .
\end{gathered}
$$

It is evident that these operators are well defined provided that Condition 2 is assumed to hold. We now define the limit coefficients as follows:

$$
\begin{gathered}
N_{p q}=Z^{p q}, \quad M_{p}=\sum_{i=1}^{m} X_{i}^{p} G_{i}, \quad L_{p}=\sum_{i=1}^{m} G_{i} Y_{i}^{p}, \\
K=i \sum_{j=1}^{r} H_{j}\left\langle\chi_{0}, \nu_{j} \chi_{0}\right\rangle+\sum_{i, j=1}^{m} G_{i} W_{i j} G_{j} .
\end{gathered}
$$

The following is the main result of this section.

Theorem 2: Suppose that Condition 2 holds and that Condition 1 holds for the limit coeffcients $N_{p q}, M_{p}, L_{p}, K$ as defined above (with $\mathcal{D}=\mathcal{H}$ ). Then

$$
\lim _{k \rightarrow \infty} \sup _{0 \leq t \leq T}\left\|R_{t}^{k * *} \psi-U_{t}^{*} \psi\right\|=0 \quad \text { for all } \psi \in \mathcal{H} \otimes \mathcal{F}, \quad T<\infty .
$$

Remark 6: We have not sought to develop this result under the most general conditions possible. In particular, the boundedness of $F_{j}, G_{j}, H_{j}$ can certainly be relaxed if appropriate domain 
assumptions are introduced, and the proof of the present result is then readily extended. We have chosen to restrict to the bounded case as the treatment of this case is particularly transparent, and we have not found one single choice of domain conditions in the unbounded case which covers all examples of interest in that setting (particularly straightforward extensions can be found when either $F_{j}=0$ for all $j$, or when only the $G_{j}$ are bounded). An illustrative example with unbounded coefficients is developed in Sec. III by appealing directly to Theorem 1.

One might worry about the well-posedness of Theorem 2 as the limit coefficients $N_{p q}, M_{p}, L_{p}, K$ depend on our choice for $\chi_{0}, \ldots, \chi_{n}$. For completeness, we provide the following simple lemma whose proof can be found in Sec. IV.

Lemma 3: Define $\chi^{k}(\alpha)$ as in Condition 2 (which we presume to be in force). Suppose that $\tilde{\chi}_{0}, \ldots, \tilde{\chi}_{n} \in \mathcal{K}$ is another orthonormal family such that for every $\alpha \in \mathbb{C}^{n}$,

$$
\tilde{\chi}^{k}(\alpha):=\tilde{\chi}_{0}+2^{-k / 2} \sum_{j=1}^{n} \alpha_{j} \tilde{\chi}_{j} \quad \text { satisfies }\left(\pi^{k} \tilde{\chi}^{k}(\alpha)\right)^{\otimes 2^{k}} \stackrel{k \rightarrow \infty}{\longrightarrow} e\left(\alpha I_{[0,1]}\right) .
$$

Then there is a $\phi \in \mathbb{R}$ such that $\tilde{\chi}_{j}=e^{i \phi} \chi_{j}$ for all $j$.

Finally, it should be noted that Condition 1 imposes stronger assumptions on the noise operators $\mu_{j}$ and $\lambda_{j}$ than is evident from Condition 2. The following lemma provides explicit assumptions which guarantee that Condition 1 holds for the coefficients $N_{p q}, M_{p}, L_{p}, K$ as defined in this section. The proof is given in Sec. IV.

Lemma 4: Define $\mathcal{S}=\operatorname{span}\left\{\chi_{1}, \ldots, \chi_{n}\right\}$, and suppose that $\mu_{j} \chi_{0} \in \mathcal{S}$ for all $j$. Suppose moreover that $\mathcal{S} \subset \operatorname{Dom}\left(\lambda_{j}\right)$ and that $\lambda_{j} \mathcal{S} \subset \mathcal{S}$ for all $j$. Then the limit coefficients $N_{p q}, M_{p}, L_{p}, K$ satisfy Condition 1.

Remark 7: It is not difficult to construct examples of discrete interaction models where the limit coefficients $N_{p q}, M_{p}, L_{p}, K$ do not satisfy the Hudson-Parthasarathy conditions in Remark 1. In this case, our proofs may be modified to show that $R_{t}^{k}$ nonetheless converges weakly to the solution $U_{t}$ of Eq. (1) with coefficients $N_{p q}, M_{p}, L_{p}, K$ as defined in this section. However, in this case the limit evolution $U_{t}$ will not be unitary, so that the physical relevance of such a result is rather limited.

\section{EXAMPLES}

We illustrate our results using four examples. The first two examples reproduce the results of Attal and Pautrat ${ }^{3}$ and Holevo. ${ }^{16}$ The third example, that of a linear quantum system, possesses both unbounded noise operators and unbounded initial coefficients. Finally, the fourth example shows that we may simultaneously approximate the noise and the initial coefficients, as is often useful in numerical simulations.

Until further notice $\mathcal{H}$ is a fixed Hilbert space. Note that for sake of example, all our models live in the Fock space with unit multiplicity $n=1$. The extension to multiple channels is entirely straightforward and leads only to complication of the notation.

\section{A. Spin chain models}

Define $\mathcal{K}=\mathbb{C}^{2}$ and denote the canonical basis of $\mathbb{C}^{2}$ as $\left(\chi_{0}, \chi_{1}\right)$. The noise Hilbert space is thus that of a single spin. We embed the spin into the Fock space by defining the embedding map $\pi^{k}: \mathcal{K} \rightarrow \mathcal{F}_{\left.2^{-k}\right]}$ through

$$
\pi^{k} \chi_{0}=e(0)=1 \oplus \bigoplus_{p=1}^{\infty} 0, \quad \pi^{k} \chi_{1}=0 \oplus 2^{k / 2} I_{\left[0,2^{-k}\right]} \oplus \bigoplus_{p=2}^{\infty} 0
$$

(here $I_{\left[0,2^{-k}\right]}$ is the indicator function on the interval $\left[0,2^{-k}\right]$ ). We now define bounded self-adjoint operators $\lambda, \mu_{1}$, and $\mu_{2}$ on $\mathcal{K}$ as matrices with respect to the canonical basis: 


$$
\lambda=\left(\begin{array}{ll}
1 & 0 \\
0 & 0
\end{array}\right), \quad \mu_{1}=\sigma_{x}=\left(\begin{array}{ll}
0 & 1 \\
1 & 0
\end{array}\right), \quad \mu_{2}=\sigma_{y}=\left(\begin{array}{cc}
0 & -i \\
i & 0
\end{array}\right) .
$$

Let $F, G_{1}, G_{2}$, and $H$ be arbitrary bounded self-adjoint operators on $\mathcal{H}$, and let $H_{K}$ be a self-adjoint operator on $\mathcal{K}$. Clearly the operator $H^{k}$ defined on $\mathcal{H} \otimes \mathcal{K}$ by

$$
H^{k}=2^{k} F \otimes \lambda+2^{k / 2}\left(G_{1} \otimes \mu_{1}+G_{2} \otimes \mu_{2}\right)+H \otimes I+I \otimes H_{K}
$$

is self-adjoint for every $k$, and the domain assumptions in Condition 2 are trivially satisfied. Furthermore, we have $\lambda \chi_{0}=0$ and $\left\langle\chi_{0}, \mu_{i} \chi_{0}\right\rangle=0$.

For every $\alpha \in \mathrm{C}$, we have defined $\chi^{k}(\alpha)=\chi_{0}+2^{-k / 2} \alpha \chi_{1}$. Note that

$$
\begin{aligned}
\left\|e\left(\alpha I_{[0,1]}\right)-\left(\pi^{k} \chi^{k}(\alpha)\right)^{\otimes 2^{k}}\right\|^{2}= & \left\|e\left(\alpha I_{\left[0,2^{-k}\right]}\right)\right\|^{k+1}+\left\|\pi^{k} \chi^{k}(\alpha)\right\|^{k^{k+1}}-\left\langle e\left(\alpha I_{\left[0,2^{-k}\right]}\right), \pi^{k} \chi^{k}(\alpha)\right\rangle^{2^{k}} \\
& -\left\langle\pi^{k} \chi^{k}(\alpha), e\left(\alpha I_{\left[0,2^{-k}\right]}\right)\right\rangle^{2^{k}}=e^{|\alpha|^{2}}-\left(1+|\alpha|^{2} 2^{-k}\right)^{2^{k}} \stackrel{k \rightarrow \infty}{\longrightarrow} 0 .
\end{aligned}
$$

We conclude that $\left(\pi^{k} \chi^{k}(\alpha)\right)^{\otimes 2^{k}} \stackrel{k \rightarrow \infty}{\longrightarrow} e\left(\alpha I_{[0,1]}\right)$. We have now verified all parts of Condition 2 . Moreover, the coefficients $N_{11}, M_{1}, L_{1}$, and $K$ are easily computed,

$$
\begin{gathered}
N_{11}=e^{i F}, \quad M_{1}=\frac{e^{i F}-I}{F}\left(G_{1}-i G_{2}\right), \quad L_{1}=\left(G_{1}+i G_{2}\right) \frac{e^{i F}-I}{F}, \\
K=i H+i\left\langle\chi_{0}, H_{K} \chi_{0}\right\rangle I+\left(G_{1}+i G_{2}\right) \frac{e^{i F}-i F-I}{F^{2}}\left(G_{1}-i G_{2}\right) .
\end{gathered}
$$

Note that these coefficients satisfy Condition 1 by virtue of Remark 1 . It follows from Theorem 2 that the repeated interaction $R_{t}^{k *}$ obtained from the Hamiltonian $H^{k}$ converges strongly to the solution $U_{t}^{*}$ of Eq. (1) with these coefficients, uniformly on compact time intervals. This result corresponds to Ref. 3, Theorem 19.

\section{B. Time-ordered exponentials}

Let $F, G, H$ be bounded operators on $\mathcal{H}$ with $F, H$ self-adjoint, and define the essentially self-adjoint operators

$$
\Delta M_{\ell}^{k}:=F\left(\Lambda_{t_{\ell}}-\Lambda_{t_{\ell-1}}\right)+G\left(A_{t_{\ell}}^{\dagger}-A_{t_{\ell-1}}^{\dagger}\right)+G^{*}\left(A_{t_{\ell}}-A_{t_{\ell-1}}\right)+H 2^{-k}
$$

on $\mathcal{H} \bar{\otimes} \mathcal{E}$, where $t_{\ell}=\ell 2^{-k}$. Holevo ${ }^{16}$ defined time-ordered stochastic exponentials as the following strong limits on $\mathcal{H} \otimes \mathcal{F}$ :

$$
\widehat{\exp }\left[-i \int_{0}^{t}\left(F d \Lambda_{s}+G d A_{s}^{\dagger}+G^{*} d A_{s}+H d s\right)\right]:=\underset{k \rightarrow \infty}{\mathrm{s}-\lim _{k \rightarrow \infty}} e^{-i \Delta M_{\left[2^{k}\right]}^{k}} \cdots e^{-i \Delta M_{1}^{k}} .
$$

Evidently this definition can be interpreted as the limit of a sequence of discrete interaction models, and the limit process does indeed solve an equation of the form (1). In this example we develop this idea by applying Theorem 2 .

Define $\mathcal{K}=\mathcal{F}_{1]}=\Gamma_{s}\left(L^{2}([0,1])\right)$, and choose the orthonormal vectors $\chi_{0}$ and $\chi_{1}$ as

$$
\chi_{0}=e(0)=1 \oplus \bigoplus_{p=1}^{\infty} 0, \quad \chi_{1}=0 \oplus I_{[0,1]} \oplus \bigoplus_{p=2}^{\infty} 0 .
$$

We define embedding maps $\pi^{k}: \mathcal{K} \rightarrow \mathcal{F}_{\left.2^{-k}\right]}$ by specifying their action on the (total) family of exponential vectors $e(f) \in \mathcal{F}_{1]}, f \in L^{2}([0,1])$ as follows: 


$$
\pi^{k} e(f)=e\left(f^{k}\right), \quad f^{k}(x)=2^{k / 2} f\left(2^{k} x\right)
$$

[note that $\left.f^{k} \in L^{2}\left(\left[0,2^{-k}\right]\right)\right]$. It is easily verified that $\pi^{k}$ is a unitary map for every $k$. We define self-adjoint operators $\mu_{1}, \mu_{2}$, and $\lambda$ on $\mathcal{K}$ as

$$
\mu_{1}=A_{1}+A_{1}^{\dagger}, \quad \mu_{2}=i\left(A_{1}-A_{1}^{\dagger}\right), \quad \lambda=\Lambda_{1},
$$

where $A_{1}, A_{1}^{\dagger}$, and $\Lambda_{1}$ are the standard Hudson-Parthasarathy noises evaluated at time $t=1$. Define $G_{1}=\left(G+G^{*}\right) / 2$ and $G_{2}=i\left(G-G^{*}\right) / 2$, and set

$$
H^{k}:=2^{k} \pi^{k *} \Delta M_{1}^{k} \pi^{k}=2^{k} F \otimes \lambda+2^{k / 2}\left(G_{1} \otimes \mu_{1}+G_{2} \otimes \mu_{2}\right)+H \otimes I .
$$

It is well known that $H^{k}$ and $\check{F}=F \otimes \lambda$ are essentially self-adjoint, and it is easily verified that $\left\langle\chi_{0}, \mu_{j} \chi_{0}\right\rangle=0$ and $\lambda \chi_{0}=0$. Moreover, as $\pi^{k} \chi_{0}$ and $\pi^{k} \chi_{1}$ coincide with their counterparts in the previous example, we have verified that Condition 2 holds. The coefficients $N_{11}, M_{1}, L_{1}$, and $K$ are now easily computed,

$$
N_{11}=e^{i F}, \quad M_{1}=\frac{e^{i F}-I}{F} G, \quad L_{1}=G^{*} \frac{e^{i F}-I}{F}, \quad K=i H+G^{*} \frac{e^{i F}-I-i F}{F^{2}} G .
$$

These coefficients satisfy Condition 1 by virtue of Remark 1. It follows from Theorem 2 that the time-ordered exponential defined above coincides with the adjoint solution $U_{t}^{*}$ of Eq. (1) with these coefficients. This agrees with Ref. 16, Corollary 1.

\section{Linear quantum systems}

Let $\mathcal{H}=\ell^{2}\left(\mathbb{Z}_{+}\right)$and denote by $\left(\eta_{k}, k \in \mathbb{Z}_{+}\right)$the canonical basis in $\ell^{2}\left(\mathbb{Z}_{+}\right)$. We also choose the domain $\mathcal{D}=\operatorname{span}\left\{\eta_{k}: k \in \mathbb{Z}_{+}\right\} \subset \mathcal{H}$ of finite particle vectors. On $\mathcal{D}$ we define the operators

$$
a \eta_{k}=\sqrt{k} \eta_{k-1}, \quad a^{*} \eta_{k}=\sqrt{k+1} \eta_{k+1}, \quad q=a+a^{*}, \quad p=i\left(a-a^{*}\right) .
$$

Note that $a$ is the annihilation operator and $a^{*}$ is the creation operator, while $q$ and $p$ are the position and momentum operators, respectively.

A linear quantum system is a QSDE of the form (1) whose coefficients take the following form on $\mathcal{D}$ :

$$
\begin{gathered}
N_{11}=I, \quad M_{1}=m p+m^{\prime} q, \quad L_{1}=-m^{*} p-m^{\prime *} q, \quad K=i H+\frac{1}{2} L_{1} M_{1}, \\
H=k_{1} p^{2}+k_{2}(p q+q p)+k_{3} q^{2}+k_{4} p+k_{5} q+k_{6} I,
\end{gathered}
$$

where $m, m^{\prime} \in \mathrm{C}$ and $k_{1}, \ldots, k_{6} \in \mathbb{R}$. Physically, a linear quantum system is a model whose Hamiltonian is quadratic in position and momentum and whose noise coefficients are linear in position and momentum. At least formally, one may easily verify that the Hudson-Parthasarathy conditions of Remark 1 are satisfied, but the coefficients are unbounded in this case. That Condition 1 is satisfied in this setting is proved in Ref. 11.

Linear quantum systems possess various special properties: for example, the adjoint solution $U_{t}^{*}$ of Eq. (1) leaves the family of Gaussian states in $\mathcal{H} \otimes \mathcal{F}$ invariant, the Heisenberg evolution of the observables $(q, p)$ has an explicit solution, and the quantum filtering problem for (1) has a finite-dimensional realization (the Kalman filter). Because of these and other properties, the linear quantum systems play a special role in quantum engineering as they admit particularly tractable methods for control synthesis and signal analysis. ${ }^{19,25,13}$ In these applications it could be of significant interest to work with discrete time approximations (e.g., for the purpose of digital signal processing), but it is important to seek approximations which preserve the linear systems properties of these models. This is easily done, but we necessarily obtain discrete approximations where 
both the initial system coefficients and the discrete noises are unbounded. In this example we will prove the convergence of such unbounded discrete approximations by appealing directly to our main theorem 1 .

We begin, however, by setting up our discrete models as in Sec. II D. Let $\mathcal{K}=\ell^{2}\left(Z_{+}\right)$, and choose an embedding $\pi^{k}: \mathcal{K} \rightarrow \mathcal{F}_{\left.2^{-k}\right]}$ by setting

$$
\pi^{k} \eta_{0}=1 \oplus \bigoplus_{p=1}^{\infty} 0, \quad \pi^{k} \eta_{\ell}=\bigoplus_{p=0}^{\ell-1} 0 \oplus\left(2^{k / 2} I_{\left[0,2^{-k}\right]}\right)^{\otimes \ell} \oplus \bigoplus_{p=\ell+1}^{\infty} 0 \quad(\ell \in \mathbb{N}) .
$$

If we define $\eta^{k}(\alpha):=\eta_{0}+2^{-k / 2} \alpha \eta_{1}$ for $\alpha \in \mathrm{C}$, then we find precisely as in the previous examples that $\left(\pi^{k} \eta^{k}(\alpha)\right)^{\otimes 2^{k}} \rightarrow e\left(\alpha I_{[0,1]}\right)$ as $k \rightarrow \infty$.

Let us now define on $\mathcal{D} \bar{\otimes} \mathcal{D}$ the operators

$$
H^{k}=H \otimes I-i\left(M_{1} \otimes a^{*}+L_{1} \otimes a\right) 2^{k / 2} .
$$

One may verify that $H^{k}$ is symmetric and that $\mathcal{D} \bar{\otimes} \mathcal{D}$ is a domain of analytic vectors for $H^{k}$, so that in particular $H^{k}$ is essentially self-adjoint for every $k$ (Ref. $28 \mathrm{Sec}$. X.6). We will subsequently identify these operators with their closures. Note that the Hamiltonian $H^{k}$ is quadratic in the family of position and momentum operators of the initial system and of the discrete noise; therefore the discrete interaction model is itself a (discrete time) linear system, and it therefore possesses all the associated desirable properties.

We now define the discrete interaction unitary $R^{k}$ on $\mathcal{H} \otimes \mathcal{F}$ from the Hamiltonian $H^{k}$ as in Sec. II D. We will use Theorem 1 to prove that

$$
\lim _{k \rightarrow \infty} \sup _{0 \leq t \leq T}\left\|R_{t}^{k *} \psi-U_{t}^{*} \psi\right\|=0 \quad \text { for all } \psi \in \mathcal{H} \otimes \mathcal{F}, \quad T<\infty,
$$

where $U_{t}$ is the solution of Eq. (1) with the coefficients $N_{11}, M_{1}, L_{1}$, and $K$ defined above. Note that Theorem 2 does not apply as the initial coefficients are unbounded, but we may essentially repeat the proof of that theorem with minor modifications to obtain the present result. To this end, we begin by noting that $\mathcal{D}$ is a core for $\mathcal{L}^{\alpha \beta}(\alpha, \beta \in \mathrm{C})$ by the analytic vector theorem (see Ref. 7, Remark 4). Let us fix $\alpha, \beta \in \mathrm{C}$ and define $\psi^{k}=\pi^{k} \eta^{k}(\alpha)$ and $\varphi^{k}=\pi^{k} \eta^{k}(\beta)$. By Theorem 1, it suffices to prove that

$$
\left\|2^{k}\left(R^{k ; \psi^{k} \varphi^{k}}-I\right) u-\mathcal{L}^{\alpha \beta} u\right\| \stackrel{k \rightarrow \infty}{\longrightarrow} 0
$$

for every $u \in \mathcal{D}$. We now proceed as follows. Fix $u \in \mathcal{D}$, and note that using the trivial identities $e^{i x}=1+f(x) x=1+i x+g(x) x^{2}$, we can write

$$
e^{i H^{k} 2^{-k}} u \otimes \eta_{\ell}=\left(I+2^{-k} f\left(H^{k} 2^{-k}\right) H^{k}\right) u \otimes \eta_{\ell}=\left(I+i H^{k} 2^{-k}+2^{-2 k} g\left(H^{k} 2^{-k}\right)\left(H^{k}\right)^{2}\right) u \otimes \eta_{\ell} .
$$

Here we have used the spectral theorem and the fact that $u \otimes \eta_{\ell} \in \operatorname{Dom}\left(\left(H^{k}\right)^{p}\right)$ for every $\ell, p$ (see the proof of Theorem 2 for a more precise argument). Therefore

$$
\begin{aligned}
\langle v \otimes & \left.\eta^{k}(\alpha), 2^{k}\left(e^{i H^{k} 2^{-k}}-I\right) u \otimes \eta^{k}(\beta)\right\rangle \\
= & i\langle v, H u\rangle-\left\langle v \otimes \eta_{0}, g\left(H^{k} 2^{-k}\right)\left(M_{1} \otimes a^{*}+L_{1} \otimes a\right) M_{1} u \otimes \eta_{1}\right\rangle-i \alpha^{*}\left\langle v \otimes \eta_{1}, f\left(H^{k} 2^{-k}\right) M_{1} u \otimes \eta_{1}\right\rangle \\
& -i\left\langle v \otimes \eta_{0}, f\left(H^{k} 2^{-k}\right)\left(M_{1} \otimes a^{*}+L_{1} \otimes a\right) u \otimes \eta_{1}\right\rangle \beta+\alpha^{*}\left\langle v \otimes \eta_{1},\left(e^{i H^{k} 2^{-k}}-I\right) u \otimes \eta_{1}\right\rangle \beta \\
& +O\left(\|v\| 2^{-k / 2}\right) .
\end{aligned}
$$

A straightforward computation shows that 


$$
\sup _{\substack{v \in \mathcal{H} \\\|v\| \leq 1}}\left|\left\langle v \otimes \eta^{k}(\alpha), 2^{k}\left(e^{i H^{k} 2^{-k}}-I\right) u \otimes \eta^{k}(\beta)\right\rangle-\left\langle v,\left(\alpha^{*} M_{1}+L_{1} \beta+K\right) u\right\rangle\right| \stackrel{k \rightarrow \infty}{\longrightarrow} 0,
$$

where we have used that $f\left(H^{k} 2^{-k}\right) \rightarrow i I, g\left(H^{k} 2^{-k}\right) \rightarrow-(1 / 2) I$, and $e^{i H^{k} 2^{-k}} \rightarrow I$ strongly as $k \rightarrow \infty$ by Ref. 29, Theorems VIII.20 and VIII.25. As in the proof of Theorem 2 below, it follows readily that $\left\|2^{k}\left(R^{k ; \psi^{k} \varphi^{k}}-I\right) u-\mathcal{L}^{\alpha \beta} u\right\| \rightarrow 0$.

\section{Finite-dimensional approximations}

In the previous examples we have approximated the QSDE (1) by constructing discrete interaction models whose interaction unitary $R^{k}$ lives on the Hilbert space $\mathcal{H} \otimes \mathcal{K}^{k}$. Even though the Fock space $\mathcal{F}$ is infinite dimensional, we have seen that we may choose finite-dimensional discrete noise spaces as simple as $\mathcal{K}^{k} \cong \mathbb{C}^{2}$. In numerical applications, however, we typically wish to go one step further and approximate also the initial space $\mathcal{H}$ (which is often infinite dimensional) by a finite-dimensional space $\mathcal{H}^{k}$. The discrete interaction models then live entirely on the finitedimensional Hilbert spaces $\mathcal{H}^{k} \otimes \mathcal{K}^{k}$, as is desirable for numerical implementation. We would like to establish that these discrete models converge to the solution of the limit equation (1) when we simultaneously let the time step go to zero and let the initial space dimension go to infinity. We will now show in a toy example that this problem fits into the setting of Theorem 1.

We will discretize the noise essentially as in the example of Sec. III A, but let us directly embed the discrete models (rather than work with the embedding maps $\pi^{k}$ ) to simplify the notation. For every $k \in \mathbb{N}$, define the vectors $\chi_{0}^{k}, \chi_{1}^{k} \in \mathcal{F}_{\left.2^{-k}\right]}$ as

$$
\chi_{0}^{k}=1 \oplus \bigoplus_{p=1}^{\infty} 0, \quad \chi_{1}^{k}=0 \oplus 2^{k / 2} I_{\left[0,2^{-k}\right]} \oplus \bigoplus_{p=2}^{\infty} 0 .
$$

Moreover, let $\mathcal{H}$ be an infinite-dimensional separable Hilbert space, and fix an orthonormal basis $\left\{\eta_{k}: k \in \mathbb{N}\right\} \subset \mathcal{H}$. For the $k$ th discrete interaction model we will choose the initial Hilbert space $\mathcal{H}^{k}:=\operatorname{span}\left\{\eta_{1}, \ldots, \eta_{k}\right\} \subset \mathcal{H}$ and the noise Hilbert space $\mathcal{K}^{k}:=\operatorname{span}\left\{\chi_{0}^{k}, \chi_{1}^{k}\right\} \subset \mathcal{F}_{2^{-k}}$. Thus $\mathcal{H}^{k} \otimes \mathcal{K}^{k}$ is indeed finite dimensional.

Let $H$ and $M$ be bounded operators on $\mathcal{H}$ where $H$ is self-adjoint. In this toy example, we will be interested in approximating the solution of the limit equation

$$
d U_{t}=U_{t}\left\{M d A_{t}^{\dagger}-M^{*} d A_{t}+i H d t-\frac{1}{2} M^{*} M d t\right\},
$$

which satisfies Condition 1 by virtue of Remark 1 . We claim that this can be done by choosing the discrete interaction unitaries

$$
R^{k}=\exp \left\{2^{-k / 2}\left(P_{k} M P_{k} \otimes b_{k}^{*}-P_{k} M^{*} P_{k} \otimes b_{k}\right)+i 2^{-k} P_{k} H P_{k} \otimes I\right\},
$$

where $P_{k}$ denotes the orthogonal projection onto $\mathcal{H}^{k}$ and the noise operator $b_{k}$ is defined by setting $b_{k} \chi_{0}^{k}=0, b_{k} \chi_{1}^{k}=\chi_{0}^{k}$, and $b_{k} \psi=0$ for $\psi \perp \mathcal{K}^{k}$.

We simply verify the conditions of Theorem 1 . As all the coefficients are bounded, we may choose $\mathcal{D}^{\alpha \beta}=\mathcal{D}=\mathcal{H}$. Fix $\alpha, \beta \in \mathrm{C}$ and $u \in \mathcal{H}$, and let us define $\psi^{k}=\chi_{0}^{k}+2^{-k / 2} \alpha \chi_{1}^{k}$ and $\varphi^{k}=\chi_{0}^{k}$ $+2^{-k / 2} \beta \chi_{1}^{k}$. As in the previous examples we have

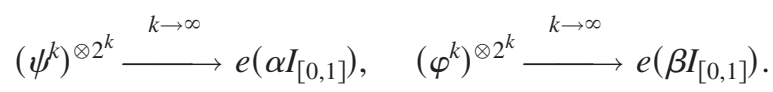

As $P_{k} u \rightarrow u$ as $k \rightarrow \infty$, it suffices to verify that

$$
2^{k}\left(R^{k ; \psi^{k} \varphi^{k}}-I\right) P_{k} u \stackrel{k \rightarrow \infty}{\longrightarrow} \mathcal{L}^{\alpha \beta} u .
$$

What remains is again essentially the same computation as in the previous example and as in the proof of Theorem 2. We leave the details to the reader. 


\section{PROOFS}

\section{A. Proof of Theorem 1}

The proof of Theorem 1 is based on a version of the Trotter-Kato theorem for contraction semigroups due to Kurtz. We cite here from Ref. 10, Theorem 1.6.5 a special case of this result in the form which will be convenient in the following.

Theorem 3: (Kurtz). Let $\mathcal{H}$ be a fixed Hilbert space. For $k \in \mathbb{N}$, let $T^{k}$ be a linear contraction on $\mathcal{H}$ and let $T_{t}$ be a strongly continuous contraction semigroup on $\mathcal{H}$ with generator $\mathcal{L}$. Let $\mathcal{D}$ be a core for $\mathcal{L}$. Then the following are equivalent.

(1) For every $u \in \mathcal{D}$, there exists $u^{k} \in \mathcal{H}$ such that

$$
u^{k} \stackrel{k \rightarrow \infty}{\longrightarrow} u, \quad 2^{k}\left(T^{k}-I\right) u^{k} \stackrel{k \rightarrow \infty}{\longrightarrow} \mathcal{L} u .
$$

(2) For every $\psi \in \mathcal{H}$ and $t<\infty$

$$
\lim _{k \rightarrow \infty}\left\|\left(T^{k}\right)^{\left\lfloor 2^{k}\right\rfloor} \psi-T_{t} \psi\right\|=0
$$

(3) For every $\psi \in \mathcal{H}$ and $t<\infty$

$$
\lim _{k \rightarrow \infty} \sup _{s \leq t}\left\|\left(T^{k}\right)^{\left\lfloor s 2^{k}\right\rfloor} \psi-T_{s} \psi\right\|=0 .
$$

Armed with this result, we may now proceed to prove theorem 1. We will first prove the forward direction, and we subsequently consider the converse implication.

Theorem 1: $(1 \Rightarrow 2)$ Let us restrict our attention to the interval $[0, N]$ with $N \in \mathbb{N}$. It suffices to prove that convergence holds uniformly on $[0, N]$ for any $N$. We may therefore restrict the Hilbert space to $\mathcal{H} \otimes \mathcal{F}_{N]}$, which we do from now on.

First, let $\alpha, \beta \in \mathbb{C}^{n}$ and $\psi^{k}, \varphi^{k} \in \mathcal{F}_{\left.2^{-k}\right]}$ as in the statement of the theorem. Then

$$
\begin{aligned}
\left\langle u \otimes\left(\psi^{k}\right)^{\otimes N 2^{k}}, R_{t}^{k} v \otimes\left(\varphi^{k}\right)^{\otimes N 2^{k}}\right\rangle & =\left\|\psi^{k}\right\|^{N 2^{k}}\left\|\varphi^{k}\right\|^{N 2^{k}}\left\langle u,\left(R^{k ; \psi^{k} \varphi^{k}}\right)^{\left.t 2^{k}\right]} v\right\rangle \stackrel{k \rightarrow \infty}{\longrightarrow} e^{\left(|\alpha|^{2}+|\beta|^{2}\right) N / 2}\left\langle u, T_{t}^{\alpha \beta} v\right\rangle \\
& =\left\langle u \otimes e\left(\alpha I_{[0, N]}\right), U_{t} v \otimes e\left(\beta I_{[0, N]}\right)\right\rangle
\end{aligned}
$$

for any $u, v \in \mathcal{H}$ and $t \in[0, N]$ by Lemma 1 and Theorem 3. Similarly, we can establish the following. Let $0=t_{0}<t_{1}<\cdots<t_{m}<t_{m+1}=N(m \in \mathbb{N})$ be a dyadic rational partition of $[0, N]$, i.e., $t_{j}=\ell_{j} 2^{-k^{\prime}}$ for some $k^{\prime} \in \mathbb{N}$ with $\ell_{j} \in \mathbb{N}$ for all $j=1, \ldots, m$. Let $\alpha_{0}, \ldots, \alpha_{m}, \beta_{0}, \ldots, \beta_{m} \in \mathbb{C}^{n}$, and choose $\psi_{j}^{k}, \varphi_{j}^{k} \in \mathcal{F}_{\left.2^{-k}\right]}$ such that

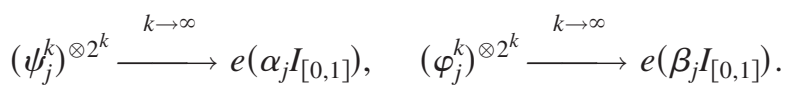

Let $f, g \in L^{2}\left([0, N] ; C^{n}\right)$ be simple functions with $f(s)=\alpha_{j}$ and $g(s)=\beta_{j}$ for $s \in\left[t_{j}, t_{j+1}[\right.$, and define for all $k \geq k^{\prime}$ the vectors

$$
\begin{aligned}
& \psi_{k}=\left(\psi_{0}^{k}\right)^{\otimes \ell_{1} 2^{k-k^{\prime}}} \otimes\left(\psi_{1}^{k}\right)^{\otimes\left(\ell_{2}-\ell_{1}\right) 2^{k-k^{\prime}}} \otimes \cdots \otimes\left(\psi_{m}^{k}\right)^{\otimes\left(N 2^{k^{\prime}}-\ell_{m}\right) 2^{k-k^{\prime}}}, \\
& \varphi_{k}=\left(\varphi_{0}^{k}\right)^{\otimes \ell_{1} 2^{k-k^{\prime}}} \otimes\left(\varphi_{1}^{k}\right)^{\otimes\left(\ell_{2}-\ell_{1}\right) 2^{k-k^{\prime}}} \otimes \cdots \otimes\left(\varphi_{m}^{k}\right)^{\otimes\left(N 2^{k^{\prime}}-\ell_{m}\right) 2^{k-k^{\prime}}} .
\end{aligned}
$$

Then $\psi_{k} \rightarrow e(f)$ and $\varphi_{k} \rightarrow e(g)$ as $k \rightarrow \infty$, and it is not difficult to verify (using the cocycle property of $U_{t}$ ) that for $t \in\left[t_{j}, t_{j+1}[\right.$

$$
\left\langle u \otimes e(f), U_{t} v \otimes e(g)\right\rangle=\|e(f)\|\|e(g)\|\left\langle u, T_{t_{1}}^{\alpha_{0} \beta_{0}} T_{t_{2}-t_{1}}^{\alpha_{1} \beta_{1}} \cdots T_{t-t_{j}}^{\alpha_{j} \beta_{j}} v .\right.
$$

We therefore obtain for $u, v \in \mathcal{H}$ and $t \in\left[t_{j}, t_{j+1}[\right.$ 


$$
\begin{aligned}
\left\langle u \otimes \psi_{k}, R_{t}^{k} v \otimes \varphi_{k}\right\rangle= & \left\|\psi_{k}\right\|\left\|\varphi_{k}\right\|\left\langle u,\left(R^{k ; \psi_{0}^{k} \varphi_{0}^{k}}\right)^{t_{1} 2^{k}} \cdots\left(R^{k ;} \psi_{j}^{k} \varphi_{j}^{k}\right)^{\left[\left(t-t_{j}\right) 2^{k}\right]} v\right\rangle \stackrel{k \rightarrow \infty}{\longrightarrow}\|e(f)\|\|e(g)\| \\
& \times\left\langle u, T_{t_{1}}^{\alpha_{0} \beta_{0}} T_{t_{2}-t_{1}}^{\alpha_{1} \beta_{1}} \cdots T_{t-t_{j}}^{\alpha_{j} \beta_{j}} v\right\rangle=\left\langle u \otimes e(f), U_{t} v \otimes e(g)\right\rangle,
\end{aligned}
$$

where we have again appealed to Lemma 1 and Theorem 3. Now note that

$$
\begin{aligned}
\mid\langle u & \left.\otimes \psi_{k}, R_{t}^{k} v \otimes \varphi_{k}\right\rangle-\left\langle u \otimes e(f), R_{t}^{k} v \otimes e(g)\right\rangle \mid \\
& \leq\left|\left\langle u \otimes\left(\psi_{k}-e(f)\right), R_{t}^{k} v \otimes \varphi_{k}\right\rangle\right|+\left|\left\langle u \otimes e(f), R_{t}^{k} v \otimes\left(\varphi_{k}-e(g)\right)\right\rangle\right| \\
& \leq\|u\|\|v\|\left(\left\|\psi_{k}-e(f)\right\|\left\|\varphi_{k}\right\|+\|e(f)\|\left\|\varphi_{k}-e(g)\right\|\right) \stackrel{{ }_{k \rightarrow \infty}}{\longrightarrow} 0,
\end{aligned}
$$

where we have used that $R_{t}^{k}$ is unitary. Therefore

$$
\left\langle u \otimes e(f), R_{t}^{k} v \otimes e(g)\right\rangle \stackrel{k \rightarrow \infty}{\longrightarrow}\left\langle u \otimes e(f), U_{t} v \otimes e(g)\right\rangle
$$

for any $u, v \in \mathcal{H}$ and simple functions $f, g \in L^{2}\left([0, N] ; \mathbb{C}^{n}\right)$ with dyadic rational jump points. As the latter is dense in $L^{2}\left([0, N] ; \mathbb{C}^{n}\right)$, a similar approximation argument shows that $R_{t}^{k}$ converges weakly to $U_{t}$ as $k \rightarrow \infty$ for every $t \in[0, N]$.

It remains to strengthen weak convergence to strong convergence uniformly on $[0, N]$. First, note that as $R_{t}^{k}$ and $U_{t}$ are all unitary, weak convergence of $R_{t}^{k}$ to $U_{t}$ [which is, of course, equivalent to weak convergence of $\left(R_{t}^{k}\right)^{*}$ to $\left.U_{t}^{*}\right]$ already implies that $\left(R_{t}^{k}\right)^{*} \rightarrow U_{t}^{*}$ strongly as $k$ $\rightarrow \infty$ for every fixed time $t \in[0, N]$. To prove that the convergence is in fact uniform, we will utilize another implication of Theorem 3.

It is convenient to extend the Fock space to two-sided time, i.e., we will consider the ampliations of all our operators to the extended Fock space $\widetilde{\mathcal{F}}=\Gamma_{s}\left(L^{2}\left(\mathbb{R} ; \mathbb{C}^{n}\right)\right) \cong \mathcal{F}_{-} \otimes \mathcal{F}$, where $\mathcal{F}_{-} \cong \mathcal{F}$ is the negative time portion of the two-sided Fock space. We now define the two-sided shift $\tilde{\theta}_{t}: L^{2}\left(\mathbb{R} ; \mathbb{C}^{n}\right) \rightarrow L^{2}\left(\mathbb{R} ; \mathbb{C}^{n}\right)$ as $\tilde{\theta}_{t} f(s)=f(t+s)$, and by $\widetilde{\Theta}_{t}: \widetilde{\mathcal{F}} \rightarrow \tilde{\mathcal{F}}$ its second quantization. Note that $\widetilde{\Theta}_{t}$ is a strongly continuous one-parameter unitary group, and that the cocycle property reads $U_{t+s}$ $=U_{s} \widetilde{\Theta}_{s}^{*} U_{t} \widetilde{\Theta}_{s}$, etc., in terms of the two-sided shift. Now define on the two-sided Fock space the operators

$$
V_{t}=\widetilde{\Theta}_{t} U_{t}^{*}, \quad S^{k}=\widetilde{\Theta}_{2^{-k}}\left(R^{k}\right)^{*} .
$$

Then it is immediate from the cocycle property that $V_{t}$ defines a strongly continuous unitary (hence contraction) semigroup on $\mathcal{H} \otimes \widetilde{\mathcal{F}}$ and that the unitary (hence contraction) $S^{k}$ is such that $\left(S^{k}\right)^{\left\lfloor 2^{k}\right]}=\widetilde{\Theta}_{2^{-k}\left[t 2^{k}\right]}\left(R_{t}^{k}\right)^{*}$. Moreover, for any dyadic rational $t$

$$
\left\|\left(R_{t}^{k}\right)^{*} \psi-U_{t}^{*} \psi\right\|=\frac{\left\|\left(S^{k}\right)^{\left[2^{k}\right]} \psi_{-} \otimes \psi-V_{t} \psi_{-} \otimes \psi\right\|}{\left\|\psi_{-}\right\|} \quad \forall \psi \in \mathcal{H} \otimes \mathcal{F}, \quad \psi_{-} \in \mathcal{F}_{-}
$$

for $k$ sufficiently large, as $\widetilde{\Theta}_{t}$ is an isometry (here $\psi_{-} \otimes \psi \in \mathcal{F}_{-} \otimes \mathcal{H} \otimes \mathcal{F} \cong \mathcal{H} \otimes \tilde{\mathcal{F}}$ ). As vectors of the form $\psi_{-} \otimes \psi$ are total in $\mathcal{H} \otimes \tilde{\mathcal{F}}$ and as we have already established that $\left\|\left(R_{t}^{k}\right)^{*} \psi-U_{t}^{*} \psi\right\| \rightarrow 0$ as $k$ $\rightarrow \infty$, we find that for every fixed dyadic rational $t$

$$
\left\|\left(S^{k}\right)^{\left\lfloor 2^{k}\right\rfloor} \psi-V_{t} \psi\right\| \stackrel{k \rightarrow \infty}{\longrightarrow} 0 \quad \text { for all } \psi \in \mathcal{H} \otimes \widetilde{\mathcal{F}} .
$$

As $V_{t}$ is strongly continuous and the dyadic rationals are dense, this evidently holds for every fixed $t$. It remains to apply the implication $2 \Rightarrow 3$ of Theorem 3 .

Theorem 1: $(2 \Rightarrow 1)$ Fix $\alpha, \beta \in \mathbb{C}^{n}, v \in \mathcal{H}$, and $t \in[0, N]$, and let us choose the sequences $\psi^{k}$ $=e\left(\alpha I_{\left[0,2^{-k}\right]}\right)$ and $\varphi^{k}=e\left(\beta I_{\left[0,2^{-k}\right]}\right)$. Then we can estimate 


$$
\begin{aligned}
\left\|\left(R^{k ; \psi^{k} \varphi^{k}}\right)^{\left.\mid t 2^{k}\right]} v-T_{t}^{\alpha \beta} v\right\|^{2} & =\sup _{\substack{u \in \mathcal{H} \\
\|u\| \leq 1}}\left|\left\langle u,\left(R^{k ; \psi^{k} \varphi^{k}}\right)^{\left.\mid t 2^{k}\right]} v-T_{t}^{\alpha \beta} v\right\rangle\right|^{2} \\
& =\sup _{\substack{u \in \mathcal{H} \\
\|u\| \leq 1}} \frac{\left|\left\langle u \otimes e\left(\alpha I_{[0, N]}\right),\left(R_{t}^{k}-U_{t}\right) v \otimes e\left(\beta I_{[0, N]}\right)\right\rangle\right|^{2}}{e^{\left(|\alpha|^{2}+|\beta|^{2}\right) N}} \\
& \leq \frac{\left\|\left(R_{t}^{k}-U_{t}\right) v \otimes e\left(\beta I_{[0, N]}\right)\right\|^{2}}{e^{|\beta|^{2} N}} \\
& =\frac{2\left\|v \otimes e\left(\beta I_{[0, N]}\right]\right\|^{2}-2 \operatorname{Re}\left(\left\langle R_{t}^{k} v \otimes e\left(\beta I_{[0, N]}\right), U_{t} v \otimes e\left(\beta I_{[0, N]}\right)\right\rangle\right)}{e^{|\beta|^{2} N}} .
\end{aligned}
$$

However by assumption $\left\|\left(R_{t}^{k *}-U_{t}^{*}\right) \psi\right\| \rightarrow 0$ as $k \rightarrow \infty$ for all $\psi \in \mathcal{H} \otimes \mathcal{F}$, so that in particular $\left\langle R_{t}^{k} v\right.$ $\left.\otimes e\left(\beta I_{[0, N]}\right), U_{t} v \otimes e\left(\beta I_{[0, N]}\right)\right\rangle \rightarrow\left\|v \otimes e\left(\beta I_{[0, N]}\right)\right\|^{2}$. We thus obtain

$$
\left\|\left(R^{k: \psi^{k} \varphi^{k}}\right)^{\left.t 2^{k}\right]} v-T_{t}^{\alpha \beta} v\right\| \stackrel{k \rightarrow \infty}{\longrightarrow} 0 \quad \text { for all } v \in \mathcal{H}, \quad t \geq 0 .
$$

The result now follows by appealing to the implication $2 \Rightarrow 1$ of Theorem 3 .

\section{B. Proof of Theorem 2}

The proof of Theorem 2 is chiefly a matter of straightforward computation. We make use of one simple trick: the trivial identities

$$
e^{i x}=1+x f(x)=1+i x+x^{2} g(x)
$$

and the conditions $\lambda_{j} \chi_{0}=0,\left\langle\chi_{0}, \mu_{j} \chi_{0}\right\rangle=0$ allow us to cancel those terms in the expression for $2^{k}\left(R^{k ; \psi^{k} \varphi^{k}}-I\right) u$ which diverge as $k \rightarrow \infty$.

Theorem 2: Throughout the proof we fix $\alpha, \beta \in \mathrm{C}^{n}$ and $u \in \mathcal{H}$, and we define $\psi^{k}=\pi^{k} \chi^{k}(\alpha)$, $\varphi^{k}=\pi^{k} \chi^{k}(\beta)$. By Theorem 1, it suffices to show that

$$
\left\|2^{k}\left(R^{k ; \psi^{k} \varphi^{k}}-I\right) u-\mathcal{L}^{\alpha \beta} u\right\| \stackrel{{ }^{k \rightarrow \infty}}{\longrightarrow} 0,
$$

where $\mathcal{L}^{\alpha \beta} u$ is given in Lemma 2 in terms of the coefficients defined in Sec. II D.

For the time being, let us fix $k$. As $H^{k}$ is self-adjoint, we may assume by the spectral theorem that $\mathcal{H} \otimes \mathcal{K} \cong L^{2}\left(\Omega^{k}\right)$ for some measure space $\left(\Omega^{k}, \Sigma^{k}, P^{k}\right)$ and that $H^{k}$ acts on $L^{2}\left(\Omega^{k}\right)$ by pointwise multiplication $\left(H^{k} \psi\right)(\omega)=h^{k}(\omega) \psi(\omega)$ for all $\psi \in L^{2}\left(\Omega^{k}\right)$. We represent the vectors $u \otimes \chi_{j}$ and $v$ $\otimes \chi_{j}$ in $L^{2}\left(\Omega^{k}\right)$ as $u_{j}(\omega)$ and $v_{j}(\omega)$, respectively. As $u \otimes \chi_{0}$ and $v \otimes \chi_{0}$ are in $\operatorname{Dom}\left(H^{k}\right)$ [so that $h^{k}(\omega) u_{0}(\omega)$ and $h^{k}(\omega) v_{0}(\omega)$ are square integrable], the trivial identity $e^{i x}=1+i x+x^{2} g(x)$ gives that

$$
\begin{aligned}
\langle v & \left.\otimes \chi_{0}, 2^{k}\left(e^{i H^{k} 2^{-k}}-I\right) u \otimes \chi_{0}\right\rangle \\
& =i \int v_{0}(\omega)^{*} h^{k}(\omega) u_{0}(\omega) P^{k}(d \omega)+2^{-k} \int\left(h^{k}(\omega) v_{0}(\omega)\right)^{*} g\left(h^{k}(\omega) 2^{-k}\right) h^{k}(\omega) u_{0}(\omega) P^{k}(d \omega) \\
& =i\left\langle v \otimes \chi_{0}, H^{k} u \otimes \chi_{0}\right\rangle+2^{-k}\left\langle H^{k} v \otimes \chi_{0}, g\left(H^{k} 2^{-k}\right) H^{k} u \otimes \chi_{0}\right\rangle .
\end{aligned}
$$

Using the trivial identity $e^{i x}=1+x f(x)$, we similarly obtain for $p=1, \ldots, n$

$$
\begin{aligned}
& \left\langle v \otimes \chi_{p}, 2^{k}\left(e^{i H^{k} 2^{-k}}-I\right) u \otimes \chi_{0}\right\rangle=\left\langle v \otimes \chi_{p}, f\left(H^{k} 2^{-k}\right) H^{k} u \otimes \chi_{0}\right\rangle, \\
& \left\langle v \otimes \chi_{0}, 2^{k}\left(e^{i H^{k} 2^{-k}}-I\right) u \otimes \chi_{p}\right\rangle=\left\langle H^{k} v \otimes \chi_{0}, f\left(H^{k} 2^{-k}\right) u \otimes \chi_{p}\right\rangle .
\end{aligned}
$$

Using that $\lambda_{j} \chi_{0}=0$ and $\left\langle\chi_{0}, \mu_{j} \chi_{0}\right\rangle=0$, a simple computation gives the following: 


$$
\begin{aligned}
\langle v \otimes & \left.\chi^{k}(\alpha), 2^{k}\left(e^{i H^{k} 2^{-k}}-I\right) u \otimes \chi^{k}(\beta)\right\rangle \\
& =i \sum_{j=1}^{r}\left\langle v \otimes \chi_{0}, H_{j} u \otimes \nu_{j} \chi_{0}\right\rangle+\sum_{j, j^{\prime}=1}^{m}\left\langle G_{j} v \otimes \mu_{j} \chi_{0}, g\left(H^{k} 2^{-k}\right) G_{j^{\prime}} u \otimes \mu_{j^{\prime}} \chi_{0}\right\rangle \\
& +\sum_{p=1}^{n} \alpha_{p}^{*} \sum_{j=1}^{m}\left\langle v \otimes \chi_{p}, f\left(H^{k} 2^{-k}\right) G_{j} u \otimes \mu_{j} \chi_{0}\right\rangle+\sum_{p=1}^{n} \sum_{j=1}^{m}\left\langle G_{j} v \otimes \mu_{j} \chi_{0}, f\left(H^{k} 2^{-k}\right) u \otimes \chi_{p}\right\rangle \beta_{p} \\
& +\sum_{p, q=1}^{n} \alpha_{p}^{*}\left\langle v \otimes \chi_{p},\left(e^{i H^{k^{2}-k}}-I\right) u \otimes \chi_{q}\right\rangle \beta_{p}+O\left(\|v\| 2^{-k / 2}\right) .
\end{aligned}
$$

To proceed, let us define

$$
\overline{\mathcal{L}}^{\alpha \beta} u=\left(\sum_{p, q=1}^{n} \alpha_{p}^{*}\left(N_{p q}-\delta_{p q}\right) \beta_{q}+\sum_{p=1}^{n} \alpha_{p}^{*} M_{p}+\sum_{p=1}^{n} L_{p} \beta_{p}+K\right) u .
$$

Using the definition of $N_{p q}, M_{p}, L_{p}, K$ in Sec. II D, we obtain

$$
\begin{aligned}
\left\langle v, \overline{\mathcal{L}}^{\alpha \beta} u\right\rangle= & i \sum_{j=1}^{r}\left\langle v \otimes \chi_{0}, H_{j} u \otimes \nu_{j} \chi_{0}\right\rangle+\sum_{j, j^{\prime}=1}^{m}\left\langle G_{j} v \otimes \mu_{j} \chi_{0}, g(\check{F}) G_{j^{\prime}} u \otimes \mu_{j^{\prime}} \chi_{0}\right\rangle \\
& +\sum_{p=1}^{n} \alpha_{p}^{*} \sum_{j=1}^{m}\left\langle v \otimes \chi_{p}, f(\check{F}) G_{j} u \otimes \mu_{j} \chi_{0}\right\rangle+\sum_{p=1}^{n} \sum_{j=1}^{m}\left\langle G_{j} v \otimes \mu_{j} \chi_{0}, f(\check{F}) u \otimes \chi_{p}\right\rangle \beta_{p} \\
& +\sum_{p, q=1}^{n} \alpha_{p}^{*}\left\langle v \otimes \chi_{p},\left(e^{i \check{F}}-I\right) u \otimes \chi_{q}\right\rangle \beta_{p} .
\end{aligned}
$$

Thus evidently we obtain

$$
\sup _{\substack{v \in \mathcal{H} \\\|v\| \leq 1}}\left|\left\langle v \otimes \chi^{k}(\alpha), 2^{k}\left(e^{i H^{k} 2^{-k}}-I\right) u \otimes \chi^{k}(\beta)\right\rangle-\left\langle v, \overline{\mathcal{L}}^{\alpha \beta} u\right\rangle\right| \stackrel{k \rightarrow \infty}{\longrightarrow} 0,
$$

provided that $f\left(H^{k} 2^{-k}\right) \rightarrow f(\check{F}), g\left(H^{k} 2^{-k}\right) \rightarrow g(\check{F})$, and $e^{i H^{k} 2^{-k}} \rightarrow e^{i \check{F}}$ strongly as $k \rightarrow \infty$. This is indeed the case due to Ref. 29, Theorems VIII.20 and VIII.25.

To complete the proof, note that we can write

$$
\begin{aligned}
\left\|2^{k}\left(R^{k ; \psi^{k} \varphi^{k}}-I\right) u-\mathcal{L}^{\alpha \beta} u\right\|= & \sup _{\substack{v \in \mathcal{H} \\
\|v\| \leq 1}}\left|\left\langle v, 2^{k}\left(R^{k ; \psi^{k} \varphi^{k}}-I\right) u\right\rangle-\left\langle v, \mathcal{L}^{\alpha \beta} u\right\rangle\right| \\
= & \sup _{\substack{v \in \mathcal{H} \\
\|v\| \leq 1}}\left|\frac{2^{k}\left\langle v \otimes \chi^{k}(\alpha), e^{i H^{k} 2^{-k}} u \otimes \chi^{k}(\beta)\right\rangle}{\left\|\chi^{k}(\alpha)\right\|\left\|\chi^{k}(\beta)\right\|}-2^{k}\langle v, u\rangle-\left\langle v, \mathcal{L}^{\alpha \beta} u\right\rangle\right| \\
\leq & \frac{\sup _{v \in \mathcal{H},\|v\| \leq 1}\left|\left\langle v \otimes \chi^{k}(\alpha), 2^{k}\left(e^{i H^{k} 2^{-k}}-I\right) u \otimes \chi^{k}(\beta)\right\rangle-\left\langle v, \overline{\mathcal{L}}^{\alpha \beta} u\right\rangle\right|}{\left\|\chi^{k}(\alpha)\right\|\left\|\chi^{k}(\beta)\right\|} \\
& +\left\|2^{k}\left(\frac{\left\langle\chi^{k}(\alpha), \chi^{k}(\beta)\right\rangle}{\left\|\chi^{k}(\alpha)\right\|\left\|\chi^{k}(\beta)\right\|}-1\right) u+\frac{\overline{\mathcal{L}^{\alpha \beta} u}}{\left\|\chi^{k}(\alpha)\right\|\left\|\chi^{k}(\beta)\right\|}-\mathcal{L}^{\alpha \beta} u\right\|,
\end{aligned}
$$

which converges to zero as $k \rightarrow \infty$. The claim has been established.

\section{Proof of Lemma 3}

By our assumptions, we have 


$$
\left\langle\chi^{k}(\alpha), \tilde{\chi}^{k}(\beta)\right\rangle^{2^{k}}=\left\langle\left(\pi^{k} \chi^{k}(\alpha)\right)^{\otimes 2^{k}},\left(\pi^{k} \tilde{\chi}^{k}(\beta)\right)^{\otimes 2^{k}}\right\rangle \stackrel{k \rightarrow \infty}{\longrightarrow} e^{\alpha^{*} \beta}
$$

for every $\alpha, \beta \in \mathbb{C}^{n}$. For $\alpha, \beta=0$ we find that $\left\langle\chi_{0}, \tilde{\chi}_{0}\right\rangle^{2^{k}} \rightarrow 1$ as $k \rightarrow \infty$, so we must have $\left|\left\langle\chi_{0}, \tilde{\chi}_{0}\right\rangle\right|$ $=1$. However $\chi_{0}$ and $\tilde{\chi}_{0}$ are unit vectors, so $\tilde{\chi}_{0}=e^{i \phi} \chi_{0}$ for some $\phi \in \mathbb{R}$ such that $e^{i \phi 2^{k}} \rightarrow 1$ as $k$ $\rightarrow \infty$. We may now compute for arbitrary $\alpha, \beta \in \mathbb{C}^{n}$

$$
\left\langle\chi^{k}(\alpha), \tilde{\chi}^{k}(\beta)\right\rangle^{2^{k}}=e^{i \phi 2^{k}}\left[1+2^{-k} e^{-i \phi} \sum_{i, j=1}^{n} \alpha_{i}^{*}\left\langle\chi_{i}, \tilde{\chi}_{j}\right\rangle \beta_{j}\right]^{2^{k}} \stackrel{k \rightarrow \infty}{\longrightarrow} \exp \left[e^{-i \phi} \sum_{i, j=1}^{n} \alpha_{i}^{*}\left\langle\chi_{i}, \tilde{\chi}_{j}\right\rangle \beta_{j}\right] .
$$

Substituting $\alpha \mapsto t \alpha$ and differentiating, we find that

$$
0=\left.\frac{d}{d t}\left[e^{t e^{-i \phi} \sum_{i, j=1}^{n} \alpha_{i}^{*}\left\langle\chi_{i}, \tilde{\chi}_{j}\right\rangle \beta_{j}}-e^{t \alpha^{*} \beta}\right]\right|_{t=0}=e^{-i \phi} \sum_{i, j=1}^{n} \alpha_{i}^{*}\left\langle\chi_{i}, \widetilde{\chi}_{j}\right\rangle \beta_{j}-\alpha^{*} \beta
$$

for every $\alpha, \beta \in \mathbb{C}^{n}$. We thus find that $\left\langle\chi_{j}, \tilde{\chi}_{j}\right\rangle=e^{i \phi}$ for every $j$, which implies that $\tilde{\chi}_{j}=e^{i \phi} \chi_{j}$ as $\chi_{j}$ and $\tilde{\chi}_{j}$ are unit vectors. The proof is complete.

\section{Proof of Lemma 4}

As the coefficients are bounded, it suffices to verify the Hudson-Parthasarathy conditions in Remark 1. We first show that

$$
\sum_{j=1}^{n} N_{p j} N_{q j}^{*}=\delta_{p q}, \quad \sum_{j=1}^{n} N_{j p}^{*} N_{j q}=\delta_{p q} .
$$

To see this, note that for all $u, v \in \mathcal{H}$, the following identities hold true:

$$
\begin{aligned}
& \langle u, v\rangle \delta_{p q}=\left\langle u \otimes \chi_{p}, e^{i \check{F}} e^{-i \check{F}} v \otimes \chi_{q}\right\rangle=\left\langle u \otimes \chi_{p}, e^{i \check{F}} P e^{-i \check{F}} v \otimes \chi_{q}\right\rangle, \\
& \langle u, v\rangle \delta_{p q}=\left\langle u \otimes \chi_{p}, e^{-i \check{F}} e^{i \check{F}} v \otimes \chi_{q}\right\rangle=\left\langle u \otimes \chi_{p}, e^{-i \check{F}} P e^{i \check{F}} v \otimes \chi_{q}\right\rangle
\end{aligned}
$$

for $p, q=1, \ldots, n$, where $P:=\sum_{r=1}^{n} I \otimes \chi_{r} \chi_{r}^{*}$ is the orthogonal projection onto $\mathcal{H} \otimes \mathcal{S}$ and we have used that $\lambda_{j} \mathcal{S} \subset \mathcal{S}$ in the last step. Using the definition of $N_{p q}$, the claim is easily established. The next condition that we will check is

$$
K+K^{*}=-\sum_{p=1}^{n} L_{p} L_{p}^{*}
$$

Since

$$
K+K^{*}=\sum_{i, j=1}^{n} G_{i}\left(W_{i j}+W_{j i}^{*}\right) G_{j}, \quad \sum_{p=1}^{n} L_{p} L_{p}^{*}=\sum_{i, j=1}^{n} G_{i}\left(\sum_{p=1}^{n} Y_{i}^{p} Y_{j}^{p *}\right) G_{j},
$$

the claim would follow immediately if we can show that for all $i$ and $j$

$$
W_{i j}+W_{j i}^{*}=-\sum_{p=1}^{n} Y_{i}^{p} Y_{j}^{p *}
$$

To see this, we begin by noting that $-f(x) f(x)^{*}=2(\cos (x)-1) / x^{2}$. Furthermore, since $\mu_{j} \chi_{0} \in \mathcal{S}$ and $\lambda_{j} \mathcal{S} \subset \mathcal{S}$, we find that $f(\check{F})^{*} v \otimes \mu_{j} \chi_{0} \in \mathcal{H} \otimes \mathcal{S}$. Therefore 


$$
f(\check{F}) f(\check{F})^{*} v \otimes \mu_{j} \chi_{0}=f(\check{F}) P f(\check{F})^{*} v \otimes \mu_{j} \chi_{0}
$$

and we can write

$$
\left\langle u \otimes \mu_{i} \chi_{0}, \frac{2 \cos (\check{F})-2 I}{\check{F}^{2}} v \otimes \mu_{j} \chi_{0}\right\rangle=-\left\langle u \otimes \mu_{i} \chi_{0}, f(\check{F}) P f(\check{F})^{*} v \otimes \mu_{j} \chi_{0}\right\rangle .
$$

Using the definition of $Y_{i}^{p}$, we find that for all $u, v \in \mathcal{H}$

$$
-\sum_{p=1}^{n}\left\langle u, Y_{i}^{p} Y_{j}^{p *} v\right\rangle=\left\langle u \otimes \mu_{i} \chi_{0}, \frac{2 \cos (\check{F})-2 I}{\check{F}^{2}} v \otimes \mu_{j} \chi_{0}\right\rangle .
$$

On the other hand, note that $g(x)+g(x)^{*}=2(\cos (x)-1) / x^{2}$. We thus obtain

$$
\left\langle u,\left(W_{i j}+W_{j i}^{*}\right) v\right\rangle=\left\langle u \otimes \mu_{i} \chi_{0}, \frac{2 \cos (\check{F})-2 I}{\check{F}^{2}} v \otimes \mu_{j} \chi_{0}\right\rangle
$$

for all $u, v \in \mathcal{H}$ from the definition of $W_{i j}$. The claim is established.

The last condition that we need to check reads

$$
M_{p}=-\sum_{q=1}^{n} N_{p q} L_{q}^{*}
$$

To see this, we begin by noting that $-e^{i x} f(x)^{*}=f(x)$. Since $\mu_{j} \chi_{0} \in \mathcal{S}$ and $\lambda_{j} \mathcal{S} \subset \mathcal{S}$, we find that $f(\check{F})^{*} G_{j} v \otimes \mu_{j} \chi_{0} \in \mathcal{S}$ for all $j=1, \ldots, m$. Therefore

$$
f(\check{F}) G_{j} v \otimes \mu_{j} \chi_{0}=-e^{i F} P f(F)^{*} G_{j} v \otimes \mu_{j} \chi_{0} .
$$

We obtain

$$
\sum_{j=1}^{m}\left\langle u \otimes \chi_{p}, f(\check{F}) G_{j} v \otimes \mu_{j} \chi_{0}\right\rangle=-\sum_{j=1}^{m}\left\langle u \otimes \chi_{p}, e^{i \check{F}} P f(\check{F})^{*} G_{j} v \otimes \mu_{j} \chi_{0}\right\rangle .
$$

Using the definitions of $N_{p q}, Y_{j}^{q}, L_{q}$, and $M_{p}$, we find that the claim holds true.

${ }^{1}$ Accardi, L., Frigerio, A., and Lewis, J. T., “Quantum stochastic processes,” Publ. Res. Inst. Math. Sci. 18, 97 (1982).

${ }^{2}$ Accardi, L., Frigerio, A., and Lu, Y. G., "The weak coupling limit as a quantum functional central limit," Commun. Math. Phys. 131, 537 (1990).

${ }^{3}$ Attal, S. and Pautrat, Y., "From repeated to continuous quantum interactions," Ann. Henri Poincare 7, 59 (2006).

${ }^{4}$ Barchielli, A., in Open Quantum Systems III: Recent Developments, edited by S. Attal, A. Joye, and C.-A. Pillet (Springer, New York, 2006), pp. 207-292.

${ }^{5}$ Bouten, L. and Silberfarb, A., "Adiabatic elimination in quantum stochastic models," Commun. Math. Phys. 283, 491 (2008).

${ }^{6}$ Bouten, L., van Handel, R., and James, M. R., “A discrete invitation to quantum filtering and feedback control," SIAM Rev. (in press).

${ }^{7}$ Bouten, L., van Handel, R., and Silberfarb, A., "Approximation and limit theorems for quantum stochastic models with unbounded coefficients," J. Funct. Anal. 254, 3123 (2008).

${ }^{8}$ Chebotarev, A. M. and Ryzhakov, G. V., "Strong resolvent convergence of the Schrödinger evolution to quantum stochastics," Math. Notes 74, 762 (2003).

${ }^{9}$ Derezinski, J. and De Roeck, W., "Extended weak coupling limit for Pauli-Fierz operators," Commun. Math. Phys. 279, 1 (2008).

${ }^{10}$ Ethier, S. N. and Kurtz, T. G., Markov Processes: Characterization and Convergence (Wiley, New York, 1986).

${ }^{11}$ Fagnola, F., "On quantum stochastic differential equations with unbounded coefficients," Probab. Theory Relat. Fields 86, 501 (1990).

${ }^{12}$ Fagnola, F., in Quantum Probability and Related Topics, edited by L. Accardi (World Scientific, Singapore, 1993), Vol. VIII, pp. 143-164.

${ }^{13}$ Geremia, J. M., Stockton, J. K., Doherty, A. C., and Mabuchi, H., "Quantum Kalman filtering and the Heisenberg limit in atomic magnetometry," Phys. Rev. Lett. 91, 250801 (2003). 
${ }^{14}$ Gough, J., "Holevo-ordering and the continuous-time limit for open floquet dynamics," Lett. Math. Phys. 67, 207 (2004).

${ }^{15}$ Gough, J., "Quantum flows as Markovian limit of emission, absorption and scattering interactions," Commun. Math. Phys. 254, 489 (2005).

${ }^{16}$ Holevo, A. S., Quantum Probability \& Related Topics, QP-PQ Vol. VII (World Scientific, River Edge, NJ, 1992), pp. 175-202.

${ }^{17}$ Holevo, A. S., "Exponential formulae in quantum stochastic calculus," Proc. R. Soc. Edinb [Biol] 126A, 375 (1996).

${ }^{18}$ Hudson, R. L. and Parthasarathy, K. R., "Quantum Itô's formula and stochastic evolutions," Commun. Math. Phys. 93, 301 (1984).

${ }^{19}$ James, M. R., Nurdin, H. I., and Petersen, I. R., " $H^{\infty}$ control of linear quantum stochastic systems," IEEE Trans. Autom. Control 53, 1787 (2008)

${ }^{20}$ Kushner, H. J., Approximation and Weak Convergence Methods for Random Processes, With Applications to Stochastic Systems Theory (MIT, Cambridge, MA, 1984).

${ }^{21}$ Lindsay, J. M. and Parthasarathy, K. R., "The passage from random walk to diffusion in quantum probability ii," Sankhya, Ser. A 50, 151 (1988)

${ }^{22}$ Lindsay, J. M. and Wills, S. J., "Construction of some quantum stochastic operator cocycles by the semigroup method," Proc. Indian Acad. Sci., Math. Sci. 116, 519 (2006).

${ }^{23}$ Lindsay, J. M. and Wills, S. J., "Quantum stochastic operator cocycles via associated semigroups," Math. Proc. Cambridge Philos. Soc. 142, 535 (2007).

${ }^{24}$ Mabuchi, H., "Coherent-feedback quantum control with a dynamic compensator," Phys. Rev. A 78, 032323 (2008).

${ }^{25}$ Nurdin, H. I., James, M. R., and Petersen, I. R., e-print arXiv:0711.2551.

${ }^{26}$ Parthasarathy, K. R., "The passage from random walk to diffusion in quantum probability," J. Appl. Probab. 25A, 151 (1988).

${ }^{27}$ Parthasarathy, K. R., An Introduction to Quantum Stochastic Calculus (Birkhäuser, Basel, 1992).

${ }^{28}$ Reed, M. and Simon, B., Fourier Analysis, Self-Adjointness, Methods of Modern Mathematical Physics Vol. 2 (Academic, New York, 1975).

${ }^{29}$ Reed, M. and Simon, B., Functional Analysis, Methods of Modern Mathematical Physics Vol. 1 (Academic, New York, 1980).

${ }^{30}$ Stroock, D. W. and Varadhan, S. R. S., Multidimensional Diffusion Processes, Grundlehren der Mathematischen Wissenschaften Vol. 233 (Springer-Verlag, Berlin, 1979). 